\title{
Investigação das propriedades magnéticas e microestrutura da ferrita de chumbo e cobre
}

\section{(Investigation of the magnetic properties and microstructure of lead and copper ferrite)}

\author{
V. A. S. Ribeiro*, G. Rodrigues, A. C. Pereira, A. F. Oliveira, C. S. P. Mendonça, \\ V. D. de Oliveira, R. C. Correa, M. R. da Silva \\ Universidade Federal de Itajubá, Av. BPS 1303, Pinheirinho, Itajubá, MG 37500-903 \\ vanderalkmin@gmail.com*,grodrigues@unifei.edu.br,amandinhafar@yahoo.com.br,adhimarflavio@unifei. \\ edu.br, sales.claudiney21@gmail.com,valescadooliveira@yahoo.com.br, robertocorrea@unifei.edu.br, \\ mrsilva@unifei.edu.br
}

\begin{abstract}
Resumo
Os objetivos deste trabalho foram a obtenção de uma ferrita de chumbo e cobre com a estrutura do espinélio, a caracterização da sua microestrutura e a determinação das suas propriedades magnéticas. A ferrita de cobre e chumbo é um material com baixa coercividade magnética, alta resistividade elétrica e alta permeabilidade magnética. Amostras com estequiometria $\mathrm{Pb}_{\mathrm{x}} \mathrm{Cu}_{1-x} \mathrm{Fe}_{2} \mathrm{O}_{4}$, $\operatorname{com}(\mathrm{x}=0,0,1 \mathrm{e} 0,2)$, foram produzidas pelo processo de metalurgia do pó e sinterizadas a $800^{\circ} \mathrm{C}$ por $6 \mathrm{~h}$ em atmosfera ambiente. As caracterizações estrutural, microestrutural e magnética foram realizadas utilizando as técnicas de difração de raios X, microscopia eletrônica de varredura, magnetômetro de amostra vibrante e análise termogravimétrica magnética. Os resultados mostram que as amostras são formadas exclusivamente pela estrutura do espinélio tetragonal. As curvas de histerese mostram características de materiais magnéticos moles e sua magnetização de saturação aumentou com a concentração de chumbo. O valor da temperatura de Curie diminuiu com o aumento da concentração de chumbo.

Palavras-chave: ferrita de chumbo, microestrutura, propriedades magnéticas, efeito Jahn Teller.
\end{abstract}

\section{Abstract}

The objectives of this work were to obtain a lead and copper ferrite with spinel structure, characterizing the microstructure and the determination of their magnetic properties. The copper and lead ferrite is a material with low magnetic coercivity, high electrical resistivity and high magnetic permeability. Samples with stoichiometry $\mathrm{Pb}_{x} \mathrm{Cu} u_{-x} \mathrm{Fe}_{2} \mathrm{O}_{4}$ with $(x=0,0.1$, and 0.2$)$ were prepared by powder metallurgy process and sintered at $800^{\circ} \mathrm{C}$ for $6 \mathrm{~h}$ in air. The structural, microstructural and magnetic characterizations were performed using the techniques of X-ray diffraction, scanning electron microscopy, vibrating sample magnetometer, and magnetic thermal gravimetric analysis. The results showed that samples are formed exclusively by the tetragonal spinel structure. Hysteresis loops show the characteristics of a soft magnetic material and the saturation magnetization increased with the lead concentration. The value of the Curie temperature decreased with increasing lead concentration.

Keywords: lead ferrites, microstructure, magnetic properties, Jahn Teller effect.

\section{INTRODUÇÃO}

A ferrita de cobre dopada com chumbo do tipo espinélio é um material com baixa coercividade magnética e alto valor de resistividade elétrica. Estas características fazem desta ferrita um excelente material na aplicação de núcleos para transformadores, absorvedor de microondas, filtros para sinal de linha, blindagem eletromagnética na área aeroespacial, sensores para a detecção de gás tóxico e gases combustíveis e capacitores com baixa permissividade [13]. As ferritas do tipo espinélio possuem fórmula química $\mathrm{MFe}_{2} \mathrm{O}_{4}$, onde $\mathrm{M}$ é um íon divalente. Os oxigênios formam uma estrutura cúbica simples compacta com 64 sítios de coordenação tetraédrica e 32 sítios de coordenação octaédrica, no qual 8 sítios de coordenação tetraédrica e 16 sítios de coordenação octaédrica são ocupados por cátions [4]. Um cátion divalente tende a ocupar os sítios tetraédricos para a formação de uma estrutura de espinélio normal; se estes cátions ocuparem os sítios octaédricos formam uma estrutura de espinélio inversa [4]. Quando estas ferritas são dopadas com cobre ocorre uma distorção na rede cristalina, ou seja, a célula unitária do espinélio sofre um discreto crescimento em uma das arestas, devido à inclusão de certa quantidade de cobre. O crescimento em uma das arestas muda a simetria do espinélio cúbico para tetragonal, sendo este efeito denominado efeito Jahn-Teller [5-8]. Neste caso a mudança da microestrutura ocorre porque o $\mathrm{Cu}^{2+}$ tem a preferência em ocupar os sítios tetraédricos ou octaédricos, enquanto que o $\mathrm{Fe}^{3+}$ passa a ocupar os sítios tetraédricos [9-13]. As propriedades magnéticas e elétricas 
destas ferritas são dependentes da composição química, do tamanho de grão e da estrutura cristalina. Por exemplo, a elevada permeabilidade magnética inicial pode ser alcançada apenas em amostras com grande tamanho de grão e com a porosidade residual localizada nos contornos de grão. Trabalhos mostram que o tamanho de grão é um dos parâmetros que mais afetam as propriedades magnéticas das ferritas, como permeabilidade magnética, correntes de Foucault, perda por histerese e resistividade elétrica. Para o aumento do tamanho de grão, a dopagem com $\mathrm{PbO}$ é uma boa escolha, pois auxilia o crescimento do grão durante a sinterização, e mesmo em baixos níveis de dopagem trazem benefícios para a densificação e consequentemente diminui a porosidade do material [14-20].

Mirzaee [18] investigou o efeito da dopagem de $\mathrm{PbO}(0$ a $3,2 \%$ em peso, com intervalos de $0,4 \%$ ) na ferrita de $\mathrm{Ni}-\mathrm{Zn}$ sinterizada a $1300{ }^{\circ} \mathrm{C}$ por $2 \mathrm{~h}$. Os resultados mostraram que a densidade aumentou de $5,15 \mathrm{~g} / \mathrm{cm}^{3}$ na amostra isenta de $\mathrm{PbO}$ para 5,30 g/ $\mathrm{cm}^{3}$ na amostra contendo $1,6 \%$ de $\mathrm{PbO}$. A partir dessa concentração houve uma diminuição na densidade para $4,98 \mathrm{~g} / \mathrm{cm}^{3}$ na amostra com $3,2 \%$ de $\mathrm{PbO}$. O aumento da densidade ocorreu devido à formação de fase líquida de $\mathrm{PbO}$ entre as partículas de ferrita; por outro lado, o aumento excessivo da espessura da camada de líquido resultou na redução da taxa de difusão e consequentemente houve uma redução na densidade. A variação da percentagem de $\mathrm{PbO}$ também causou variação no tamanho de grão que aumentou de $1,8 \mu \mathrm{m}$ para a amostra isenta de $\mathrm{PbO}$ para $17,1 \mu \mathrm{m}$ na amostra contendo $2,4 \%$ de $\mathrm{PbO}$. A partir dessa concentração observou-se uma diminuição no tamanho de grão para $6,4 \mu \mathrm{m}$ na amostra contendo $3,2 \%$ de $\mathrm{PbO}$. A variação do tamanho de grão é evidenciada em três faixas de dopagem distintas. A primeira, em concentrações de 0 a $0,4 \%$ em peso de $\mathrm{PbO}$, ocorre a difusão dos átomos constituintes na estrutura da ferrita de Ni-Zn. Isto ocorre devido a ausência ou da presença de pequenas quantidades da fase líquida nos contornos de grão. Em concentrações com 0,4 a 2,4\% em peso ocorre a difusão dos átomos através da fase líquida rica em $\mathrm{PbO}$. Esta faixa de concentação é caracterizada pelo rápido crescimento de grãos e considerável diminuição da energia de ativação. Em concentrações maiores que 2,4\% em peso, ocorre a difusão através da camada de líquido obtida pela fusão do $\mathrm{PbO}$. A grande quantidade de $\mathrm{PbO}$ aumenta a espessura da camada da fase líquida. O crescimento dos grãos e a energia de ativação depende da espessura da camada de difusão e o tamanho de grão diminui gradualmente com o aumento da percentagem de $\mathrm{PbO}$ que aumenta a espessura da camada de difusão. Com relação à temperatura de Curie observou-se que a variação na percentagem de $\mathrm{PbO}$ não causou alterações significativas, mantendo valores entre 233 e $252{ }^{\circ} \mathrm{C}$. A magnetização de saturação diminuiu de 78,3 emu/g para a ferrita isenta de $\mathrm{PbO}$ para $71,2 \mathrm{emu} / \mathrm{g}$ para a ferrita com 3,2\% em peso de $\mathrm{PbO}$ [18].

Ullah et al. [17] investigaram o efeito da dopagem de $\mathrm{Pb}$ em ligas com composições $\mathrm{Sr}_{1-\mathrm{x}} \mathrm{Pb}_{\mathrm{x}} \mathrm{Fe}_{12} \mathrm{O}_{19}(\mathrm{x}=0,00 ; 0,05$; $0,10 ; 0,15$ e 0,2$)$ sinterizadas a $800{ }^{\circ} \mathrm{C}$ por $2 \mathrm{~h}$. Os resultados mostraram que a dopagem com $\mathrm{Pb}$ na ferrita de estrôncio afetaram consideravelmente a magnetização de saturação, campo coercivo e remanência. A magnetização de saturação diminuiu de 45,80 $\pm 0,75 \mathrm{emu} / \mathrm{g}$ para a ferrita isenta de chumbo $(\mathrm{x}=0)$ para $24,60 \pm 0,60 \mathrm{emu} / \mathrm{g}$ na ferrita com estequiometria $\mathrm{x}=0,20$ e a remanência diminuiu de 22,80 $\pm 0,60$ para a ferrita isenta de chumbo para $14,80 \pm 0,40$ $\mathrm{emu} / \mathrm{g}$ na ferrita com estequiometria $\mathrm{x}=0,2$. A coercividade aumentou de $2280 \pm 40$ Oe para a ferrita isenta de chumbo $(\mathrm{x}=0)$ para $3820 \pm 40$ Oe na ferrita com estequiometria $\mathrm{x}=$ 0,20. A diminuição da magnetização de saturação ocorreu devido à substituição de um material paramagnético $\left(\mathrm{Sr}^{2+}\right)$ por um diamagnético $\left(\mathrm{Pb}^{2+}\right)$. Haq e Anis-ur-Rehman [21] investigaram o efeito da dopagem de $\mathrm{Pb}$ em ligas com composições $\mathrm{Ba}_{1-\mathrm{x}} \mathrm{Pb}_{\mathrm{x}} \mathrm{Fe}_{12} \mathrm{O}_{19}(\mathrm{x}=0,0 ; 0,2 ; 0,4 ; 0,6 ; 0,8$ e 1,0) sinterizadas a $965^{\circ} \mathrm{C}$ por $3 \mathrm{~h}$. Os resultados mostraram que o campo coercivo aumentou de 5,34 Oe para a ferrita isenta de chumbo $(x=0)$ para 5,39 Oe para a ferrita com estequiometria $\mathrm{x}=0,2$. A partir desta concentração houve uma diminuição para 2,72 Oe das amostras com estequiometrias com $\mathrm{x}=1$. O campo coercivo diminuiu devido ao movimento limitado nas paredes dos domínios e aumento de tamanho de grãos. Gaikwad [1] investigou a influência da dopagem de $\mathrm{PbO}$ (0,5\% em peso) em ferritas de cobre sinterizadas a 960 ${ }^{\circ} \mathrm{C}$ por $8 \mathrm{~h}$. Os resultados mostraram que a magnetização de saturação diminuiu de 15,839 emu/g na amostra isenta de chumbo para 11,646 emu/g na amostra com $0,5 \%$ em peso de $\mathrm{PbO}$ e o campo coercivo diminuiu de $983 \mathrm{G}$ para a amostra isenta de chumbo para $963,49 \mathrm{G}$ na amostra com $0,5 \%$ em peso de $\mathrm{PbO}$.

Sachelarie et al. [22] investigaram o efeito da dopagem de $\mathrm{PbO}(1,2 \%$ em peso) nas ferritas de NiZn sinterizadas a $1100{ }^{\circ} \mathrm{C}$ por $4 \mathrm{~h}, \mathrm{LiZn}$ sinterizadas a $1050{ }^{\circ} \mathrm{C}$ por $4 \mathrm{~h} \mathrm{e}$ $\mathrm{MgZnCu}$ sinterizadas a $1050{ }^{\circ} \mathrm{C}$ por $4 \mathrm{~h}$. Seus resultados mostraram que a densidade das amostras NiZn aumentou de $4,1 \mathrm{~g} / \mathrm{cm}^{3}$ para aquela isenta de $\mathrm{PbO}$ para $5 \mathrm{~g} / \mathrm{cm}^{3}$ para a amostra contendo $1,2 \%$ de $\mathrm{PbO}$. Observou-se também um aumento na densidade das amostras de LiZn de 3,9 g/ $\mathrm{cm}^{3}$ para a amostra isenta de $\mathrm{PbO}$ para $4,7 \mathrm{~g} / \mathrm{cm}^{3}$ para a amostra contendo $1,2 \%$ de $\mathrm{PbO}$. A densidade das amostras de $\mathrm{MgZnCu}$ aumento de $4,2 \mathrm{~g} / \mathrm{cm}^{3}$ para a amostra isenta de $\mathrm{PbO}$ para $4,8 \mathrm{~g} / \mathrm{cm}^{3}$ para a amostra de $\mathrm{MgZnCu}$ contendo $1,2 \%$ de $\mathrm{PbO}$. A dopagem de $1,2 \%$ de $\mathrm{PbO}$ nas ferritas também causou um aumento no tamanho de grão nas amostras de NiZn de $0,8 \mu \mathrm{m}$ para a amostra isenta de $\mathrm{PbO}$ para $2 \mu \mathrm{m}$ na amostra contendo $1,2 \%$ de $\mathrm{PbO}$. O tamanho de grão das amostras LiZn também aumentou de $1 \mu \mathrm{m}$ para a amostra isenta de $\mathrm{PbO}$ para $20 \mu \mathrm{m}$ na amostra contendo $1,2 \% \mathrm{PbO}$. Comportamento similar foi observado para as amostras de $\mathrm{MgZnCu}$; nestas o tamanho de grão aumentou de $3 \mu \mathrm{m}$ para a amostra isenta de $\mathrm{PbO}$ para $12 \mu \mathrm{m}$ nas amostras contendo $1,2 \% \mathrm{PbO}$. A dopagem de $1,2 \%$ de $\mathrm{PbO}$ em peso nas ferritas também causou um aumento na magnetização de saturação de 51,5 emu/g para a amostra de NiZn isenta de $\mathrm{PbO}$ para 56 emu/g para a amostra de NiZn dopada com 1,2\% PbO. Nas amostras de LiZn a magnetização de saturação aumentou de $72 \mathrm{emu} / \mathrm{g}$ para a amostra isenta de $\mathrm{PbO}$ para $74,7 \mathrm{emu} / \mathrm{g}$ na contendo $1,2 \% \mathrm{PbO}$. Nas amostras de $\mathrm{MgZnCu}$, aumentou 
de 57,5 emu/g para a amostra isenta de $\mathrm{PbO}$ para 60,2 $\mathrm{emu} / \mathrm{g}$ para a amostra contendo 1,2\% PbO. Observouse que a magnetização de saturação não teve variação significativa com a dopagem de $\mathrm{PbO}$. A presença dos poros intragranulares ou o efeito da não-homogeneidade nos grãos justificaram os resultados obtidos [22]. Rezlescu et al. [14] investigaram o efeito da dopagem de $\mathrm{PbO}(0,3$ a $4 \%$ em peso, com intervalos de $0,3 \%$ ) na ferrita de $\mathrm{Ni}-\mathrm{Zn}$ sinterizada de 800 a $1200{ }^{\circ} \mathrm{C}$ com intervalos de $100{ }^{\circ} \mathrm{C}$, durante $3 \mathrm{~h}$. Seus resultados monstraram que o $\mathrm{PbO}$ teve um grande impacto sobre a densidade e a magnetização de saturação da ferrita de NiZn. Para as amostras sinterizadas a $1000{ }^{\circ} \mathrm{C}$, a densidade aumentou de $3,2 \mathrm{~g} / \mathrm{cm}^{3}$ para a amostra com $0,6 \%$ em peso de $\mathrm{PbO}$ para $4 \mathrm{~g} / \mathrm{cm}^{3}$ para a amostra contendo $4,2 \%$ em peso de PbO. Em 1100 e $1200{ }^{\circ} \mathrm{C}$, a densidade atingiu seus valores máximos de $4,9 \mathrm{~g} / \mathrm{cm}^{3}$ e $5,05 \mathrm{~g} / \mathrm{cm}^{3}$, respectivamente, para as amostras dopadas com $1,2 \%$ de $\mathrm{PbO}$ em peso. Acima desse nível de dopagem, a diminuição da densidade foi atribuída ao aumento da porosidade intragranular, resultado do crescimento de grão descontínuo. Observou-se que nas amostras dopadas entre $0,9 \%$ e $1,5 \%$ em peso de $\mathrm{PbO}$ houve um aumento da magnetização de saturação em consequência da estrutura de empacotamento denso das partículas [14]. Os objetivos deste trabalho foram a obtenção de uma ferrita de chumbo e cobre com a estrutura do espinélio de estequiometria $\mathrm{Pb}_{\mathrm{x}} \mathrm{Cu}_{1-\mathrm{x}} \mathrm{Fe}_{2} \mathrm{O}_{4}(\mathrm{x}=0 ; 0,1 ; 0,2)$, preparada pelo processo de tecnologia do pó, e a caracterização microestrutural e magnética das amostras sinterizadas.

\section{MATERIAIS E MÉTODOS}

Amostras com estequiometria $\mathrm{Pb}_{\mathrm{x}} \mathrm{Cu}_{1-\mathrm{x}} \mathrm{Fe}_{2} \mathrm{O}_{4}(\mathrm{x}=0$; $0,10 ; 0,20)$ foram preparadas pelo processo de metalurgia do pó utilizando óxidos de alta pureza de $\mathrm{PbO}$ (min. 99,9\% em peso), $\mathrm{CuO}$ (min. 99,9\% em peso) e $\mathrm{Fe}_{2} \mathrm{O}_{3}$ (min. 99,9\% em peso). As amostras foram compactadas em uma prensa uniaxial e sinterizados a $800{ }^{\circ} \mathrm{C} / 6 \mathrm{~h}$ em atmosfera ambiente. A caracterização estrutural foi realizada pelas as técnicas de difração de raios X (DRX) e microscopia eletrônica de varredura (MEV). A DRX foi realizada utilizando-se um difratômetro da Panalytical, $\mathrm{X}^{\prime}$ pert $\mathrm{MPD}$, com radiação $\mathrm{Cu}-\mathrm{K} \alpha$, varredura de $15^{\circ}$ a $80^{\circ}$, passo de $0,05^{\circ}$ e tempo de contagem de $2 \mathrm{~s}$ por ponto. As medidas de microscopia eletrônica de varredura foram realizadas utilizando um equipamento da Zeiss, EVO MA15. O refinamento das estruturas cristalinas foram realizados utilizando o software FullProff. Os parâmetros magnéticos foram determinados através das curvas de histerese magnética. Os ciclos de histerese magnética foram obtidos através de um magnetômetro de amostra vibrante com um campo aplicado até $14 \mathrm{kG}$. A identificação da temperatura de Curie foi realizada acoplando um ímã em um analisador termogravimétrico da Shimadzu, TGA-50, entre a temperatura ambiente e $1000{ }^{\circ} \mathrm{C}$ com uma taxa de aquecimento de $10^{\circ} \mathrm{C} / \mathrm{min}$. A determinação do tamanho de grão foi feita utilizando-se o programa de processamento digital Image $\mathrm{J}$, onde foi realizada a contagem média de no mínimo de 1000 grãos por amostra. Os valores da densidade a verde das amostras não sinterizadas foram obtidos pelo método geométrico (massa por volume) e a densidade aparente das amostras sinterizadas foram determinadas pelo método de imersão utilizando o princípio de Arquimedes (norma ABNT-NBR 6620). A porosidade aparente é definida como o quociente do volume dos poros abertos pelo volume aparente, calculado pelo volume do material sólido mais o volume dos poros abertos e fechados. A densidade aparente foi calculada pela Equação (A) [23-25]:

$$
\mathrm{Da}=\left(\frac{\mathrm{ms}}{\mathrm{mu}-\mathrm{mi}}\right) \mathrm{D}_{\mathrm{H}_{2} \mathrm{O}}
$$

onde: $m s$ foi a massa seca da amostra em g, $m u$ foi a massa úmida da amostra em $\mathrm{g}$, após ela ter permanecido imersa em água durante $24 \mathrm{~h}, m i$ foi a massa da amostra imersa em g em água e $D_{H 20}$ foi a densidade da água na temperatura em que foi realizada a medida $\mathrm{em} \mathrm{g} / \mathrm{cm}^{3}$. Através deste método, foi possível calcular a porosidade aparente $\mathrm{Pa}$ a partir da Equação (B) [23, 24]:

$$
\mathrm{Pa}=\left(\frac{\mathrm{mu}-\mathrm{ms}}{\mathrm{mu}-\mathrm{mi}}\right)
$$

\section{RESULTADOS E DISCUSSÃO}

A Fig. 1 mostra os difratogramas de raios $\mathrm{X}$ das amostras com diferentes estequiometrias $(\mathrm{x}=0 ; 0,10$; $0,20)$, sinterizadas a $800{ }^{\circ} \mathrm{C}$ por $6 \mathrm{~h}$. Pode-se observar que as amostras são formadas exclusivamente pela fase ferrita com estrutura de espinélio com simetria tetragonal (JCPDS 96-901-1013). Essa transformação da estrutura cúbica para tetragonal no espinélio é uma evidência que as amostras estão sofrendo o efeito Jahn-Teller, que também pode ser

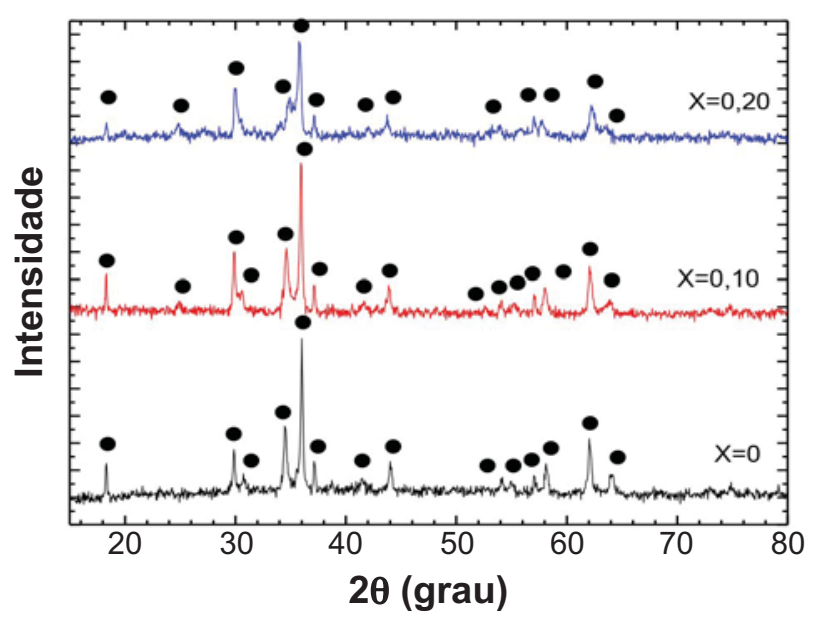

Figura 1: Difratogramas de raios $\mathrm{X}$ das amostras sinterizadas de $\mathrm{Pb}_{\mathrm{x}} \mathrm{Cu}_{(1-\mathrm{x})} \mathrm{Fe}_{2} \mathrm{O}_{4}(\mathrm{x}=0,0,1,0,2)$.

[Figure 1: XRD patterns of sintered samples of $\mathrm{Pb}_{x} \mathrm{Cu}_{(1-x)} \mathrm{Fe}_{2} \mathrm{O}_{4}$ $(x=0,0.1,0.2)$.] 
encontrado em altas concentrações de $\mathrm{Cu}(\mathrm{x}=0$ a 0,3$)$ [26]. Através do refinamento dos parâmetros da estrutura cristalina observou-se uma variação dos parâmetros de rede em função da percentagem de $\mathrm{PbO}$ (Tabela I). O aumento do parâmetro de rede $a$ em função da percentagem de chumbo é atribuído ao tamanho do raio iônico do $\mathrm{Pb}^{2+}(1,19 \AA)$ ser maior do que o raio ionico do $\mathrm{Cu}^{2+}(0,89 \AA)$ [26].

A Fig. 2 mostra as curvas de histerese das amostras de $\mathrm{Pb}_{\mathrm{x}} \mathrm{Cu}_{1-\mathrm{x}} \mathrm{Fe}_{2} \mathrm{O}_{4}$ com diferentes estequiometrias. Observa-se que estas curvas possuem características de um material magnético mole, comportamento este que foi atribuído à estreita área interna dessas curvas. Este comportamento também pode ser atribuído pelos valores menores que 1000 Oe do campo coercivo $\left(\mathrm{H}_{\mathrm{c}}\right)$ (Tabela II). As curvas de histerese obtidas neste trabalho possuem as mesmas características e comportamento das curvas obtidas por Gaikwad, para amostras isentas de chumbo [1].

A Fig. 3 mostra as curvas de magnetização em função de temperatura. Os resultados da temperatura de Curie diminuíram de $348,53{ }^{\circ} \mathrm{C}$ na ferrita isenta de chumbo $(\mathrm{x}=$ 0) para $347,27^{\circ} \mathrm{C}$ na ferrita com $\mathrm{x}=0,2$. A diminuição da temperatura de Curie em função da percentagem de chumbo ocorreu devido à substituição do $\mathrm{Pb}^{2+}$ (diamagnético) por $\mathrm{Cu}^{2+}$ (paramagnético). A variação da temperatura de Curie é visualizada em alguns trabalhos com ferritas de $\mathrm{Mg}-\mathrm{Zn}$ [27], Li-Zn [28], Ni-Cd [29], Co-Cd [30], e Li-Cd [31].

Tabela I - Parâmetros de rede do $\mathrm{Pb}_{\mathrm{x}} \mathrm{Cu}_{(1-\mathrm{x})} \mathrm{Fe}_{2} \mathrm{O}_{4}$ calculados por meio do software Fullproof.

[Table I - Lattice constants $(a, b, c)$ of $\mathrm{Pb}_{x} \mathrm{Cu}_{(1-x)} \mathrm{Fe}_{2} \mathrm{O}_{4}$ calculated by Fullproof software.]

\begin{tabular}{cccc}
\hline Amostra & $a(\AA)$ & $b(\AA)$ & $c(\AA)$ \\
\hline $\mathrm{X}=0$ & $5,81 \pm 0,01$ & $5,81 \pm 0,01$ & $8,70 \pm 0,01$ \\
$\mathrm{X}=0,10$ & $5,83 \pm 0,01$ & $5,83 \pm 0,01$ & $8,69 \pm 0,01$ \\
$\mathrm{X}=0,20$ & $5,86 \pm 0,01$ & $5,86 \pm 0,01$ & $8,58 \pm 0,01$ \\
\hline
\end{tabular}

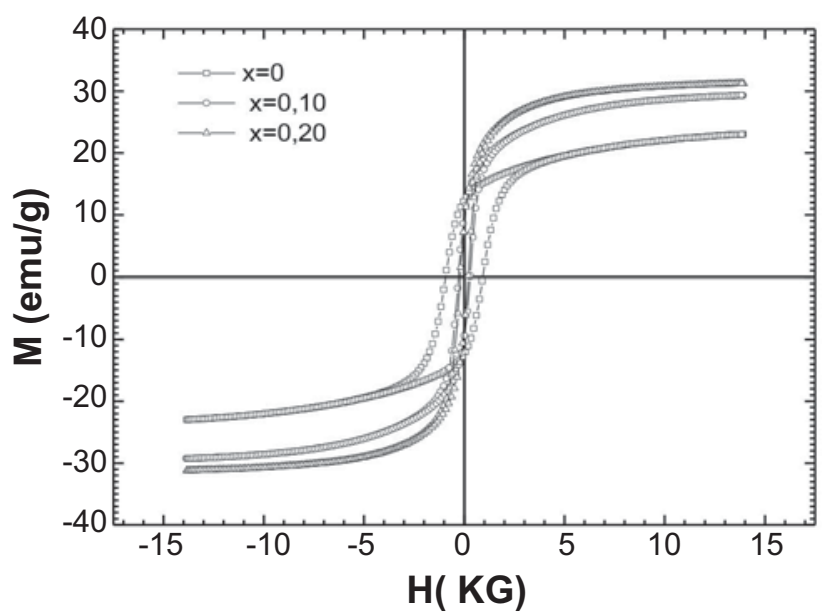

Figura 2: Ciclo de histerese M-H. $(\square) \mathrm{x}=0,(\mathrm{o}) \mathrm{x}=0,10$ e $(\Delta) \mathrm{x}$ $=0,20$.

[Figure 2: Hysteresis loop M-H. ( $\square$ ) $x=0$, (o) $x=0.10$ and $(\Delta)$ $x=0.20$.]

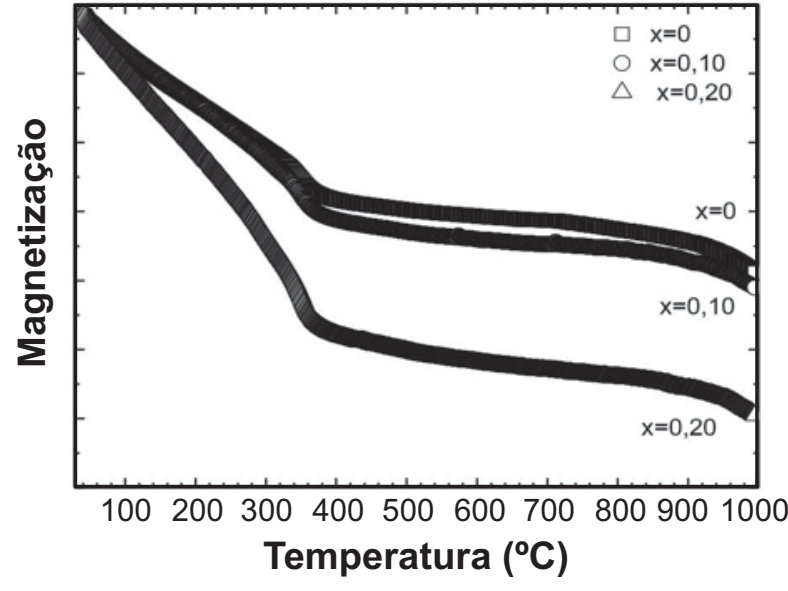

Figura 3: Magnetização dependente da temperatura de diferentes amostras. $(\square) \mathrm{x}=0,(\mathrm{o}) \mathrm{x}=0,10$ e $(\Delta) \mathrm{x}=0,20$.

[Figure 3: Temperature dependence of the magnetization for the different samples. $(\square) x=0$, (o) $x=0.10$ and $(\Delta) x=0.20$.]

A Tabela II mostra os resultados da magnetização de saturação $(\mathrm{Ms})$, campo coercivo $\left(\mathrm{H}_{\mathrm{C}}\right)$, magnetização remanente $\left(\mathrm{M}_{\mathrm{R}}\right)$ e temperatura de Curie $(\mathrm{Tc})$. Os resultados da magnetização de saturação aumentaram de $23 \mathrm{emu} / \mathrm{g}$ para a ferrita isenta de chumbo $(\mathrm{x}=0)$ para $31,19 \mathrm{emu} / \mathrm{g}$ para a ferrita contendo $\mathrm{x}=0,2$. Segundo Torquato et al. [4], o aumento da magnetização de saturação ocorre devido à facilidade do deslocamento reversível da parede dos domínios e para ocorrer esse deslocamento é necessário que a energia do campo externo aplicado seja maior que a energia de fixação da parede. Assim, quanto maior o tamanho de grão, menor será a área de contorno de grão e mais fácil será o deslocamento da parede de domínios na direção do campo magnético aplicado; consequentemente, maior será a magnetização [4]. Os resultados da magnetização remanente diminuíram de $12,33 \mathrm{emu} / \mathrm{g}$ para a ferrita isenta de chumbo $(\mathrm{x}=0)$ para $8,21 \mathrm{emu} / \mathrm{g}$ para a ferrita com estequiometria $\mathrm{x}$ $=0,20$. A diminuição da magnetização remanente ocorreu devido à substituição do $\mathrm{Pb}^{2+}$ (diamagnético) pelo $\mathrm{Cu}^{2+}$ (paramagnético) [32]. Os resultados do campo coercivo diminuíram de $922,52 \mathrm{G}$ para a ferrita isenta de chumbo $(\mathrm{x}=0)$ para $203,73 \mathrm{G}$ para a ferrita com estequiometria $\mathrm{x}$ $=0,20$. A diminuição do campo coercivo ocorreu devido à dependência do campo coercivo com o tamanho do grão, ou seja, quanto maior o tamanho do grão, menor é o campo coercivo $[18,22]$. Comparando os resultados da magnetização de saturação obtidos neste trabalho com os resultados obtidos por Gaikwad [1], observa-se que o valor da magnetização de saturação de $23 \mathrm{emu} / \mathrm{g}$ para a ferrita isenta de chumbo $(\mathrm{x}=0)$ foi maior que a magnetização de saturação de 11,646 emu/g para a ferrita isenta de chumbo obtida por [1]. Observou-se também que o resultado do campo coercivo de $922,52 \mathrm{G}$ para a ferrita isenta de chumbo $(\mathrm{x}=0)$ está próximo ao resultado obtido por [1], que foi de $963,49 \mathrm{G}$.

A Tabela III mostra os resultados da densidade a verde $\left(D_{v}\right)$ para as amostras não sinterizadas e densidade aparente $\left(D_{\mathrm{a}}\right)$ e porosidade aparente $\left(\mathrm{P}_{\mathrm{a}}\right)$ para as amostras 
Tabela II - Valores da magnetização de saturação $\left(\mathrm{M}_{\mathrm{s}}\right)$, coercividade $\left(\mathrm{H}_{\mathrm{c}}\right)$, magnetização remanente $\left(\mathrm{M}_{\mathrm{r}}\right)$, e temperatura de Curie (Tc).

[Table II - Values of saturation magnetization (Ms), coercive field $(\mathrm{Hc})$, remanent magnetization $(\mathrm{Mr})$, and Curie temperature (Tc).]

\begin{tabular}{ccccc}
\hline Amostras & $\mathrm{M}_{\mathrm{S}}(\mathrm{emu} / \mathrm{g})$ & $\mathrm{H}_{\mathrm{C}}(\mathrm{G})$ & $\mathrm{M}_{\mathrm{R}}(\mathrm{emu} / \mathrm{g})$ & $\mathrm{Tc}\left({ }^{\circ} \mathrm{C}\right)$ \\
\hline $\mathrm{x}=0$ & 23,00 & 922,52 & 12,33 & 348,53 \\
$\mathrm{x}=0,10$ & 29,23 & 289,77 & 9,75 & 348,48 \\
$\mathrm{x}=0,20$ & 31,19 & 203,73 & 8,21 & 347,27 \\
\hline
\end{tabular}

sinterizadas a $800{ }^{\circ} \mathrm{C} / 6 \mathrm{~h}$. Os valores da densidade a verde aumentaram de $1,15 \mathrm{~g} / \mathrm{cm}^{3}$ para a ferrita isenta de chumbo $(\mathrm{x}=0)$ para $3,03 \mathrm{~g} / \mathrm{cm}^{3}$ para a ferrita contendo $\mathrm{x}$ $=0,20$. Os resultados $\left(\mathrm{D}_{\mathrm{v}} / \mathrm{D}_{\mathrm{t}}\right)$ foram superiores a $50 \%$, o que mostra que em todos os sistemas investigados houve uma boa compactação das mesmas, exceto para a ferrita isenta de chumbo $(x=0)$ observou-se valor de $(\mathrm{Dv} / \mathrm{Dt}) \mathrm{de}$ 21,33\%. Após a sinterização observou-se que a densidade aparente, aumentou de $3,07 \mathrm{~g} / \mathrm{cm}^{3}$ para a ferrita isenta de chumbo $(\mathrm{x}=0)$ para $3,70 \mathrm{~g} / \mathrm{cm}^{3}$ para a ferrita contendo $\mathrm{x}$ $=0,20$ mostrando que seus valores de densidade alcançados foram muito baixos. Observou-se também que houve uma diminuição na porosidade aparente de $14,0 \%$ para a ferrita isenta de chumbo para $10,6 \%$ para a ferrita contendo $\mathrm{x}=$ 0,20 . Os valores da densidade relativa $\left(D_{a} / D_{t}\right)$ aumentaram com a concentração de chumbo, foram de $56,95 \%$ para a ferrita isenta de chumbo $(\mathrm{x}=0)$ para $68,64 \%$ para a ferrita contendo $\mathrm{x}=0,20$, mostrando a influência do $\mathrm{PbO}$ no processo de sinterização ( $\mathrm{Da} / \mathrm{Dt}>60 \%)$. De uma maneira geral, o aumento da concentração de chumbo contribuiu para a diminuição da porosidade intergranular (no contorno de grão), pois sua ausência facilita o deslocamento reversível das paredes dos domínios na direção do campo magnético aplicado, sendo um dos fatores que também contribuiu para o aumento da magnetização. Segundo [33, 34], a porosidade afeta o processo de magnetização, porque os poros trabalham como gerador de um campo de desmagnetização,

Tabela III - Resultados de densidade a verde $\left(\mathrm{D}_{\mathrm{V}}\right)$ para as amostras não sinterizadas e densidade aparente $\left(D_{\mathrm{a}}\right)$ e porosidade aparente $\left(\mathrm{P}_{\mathrm{a}}\right)$ para as amostras sinterizadas a $800{ }^{\circ} \mathrm{C} / 6 \mathrm{~h}$ com taxa de aquecimento de $10{ }^{\circ} \mathrm{C} / \mathrm{min}$.

[Table III - Results of green density $\left(D_{V}\right)$ for the nonsintered samples and apparent density $\left(D_{d}\right)$ and apparent porosity $\left(P_{d}\right)$ for the samples sintered at $800^{\circ} \mathrm{C} / 6 \mathrm{~h}$ with heating rate of $10{ }^{\circ} \mathrm{C} / \mathrm{min}$.]

\begin{tabular}{cccccc}
\hline $\mathrm{x}$ & $\begin{array}{c}\mathrm{D}_{\mathrm{v}} \\
\left(\mathrm{g} / \mathrm{cm}^{3}\right)\end{array}$ & $\begin{array}{c}\mathrm{D}_{\mathrm{v}} / \mathrm{D}_{\mathrm{t}} \\
(\%)\end{array}$ & $\begin{array}{c}\mathrm{D}_{\mathrm{a}} \\
\left(\mathrm{g} / \mathrm{cm}^{3}\right)\end{array}$ & $\begin{array}{c}\mathrm{D}_{\mathrm{a}} / \mathrm{D}_{\mathrm{t}} \\
(\%)\end{array}$ & $\begin{array}{c}\mathrm{P}_{\mathrm{a}} \\
(\%)\end{array}$ \\
\hline 0 & 1,15 & 21,3 & 3,07 & 57,0 & 14,0 \\
0,1 & 2,78 & 51,6 & 3,41 & 63,2 & 12,2 \\
0,2 & 3,03 & 56,2 & 3,70 & 68,7 & 10,6 \\
\hline
\end{tabular}

Densidade teórica (Dt): 5,39 g/cm (JCPDS 96-901-1013).
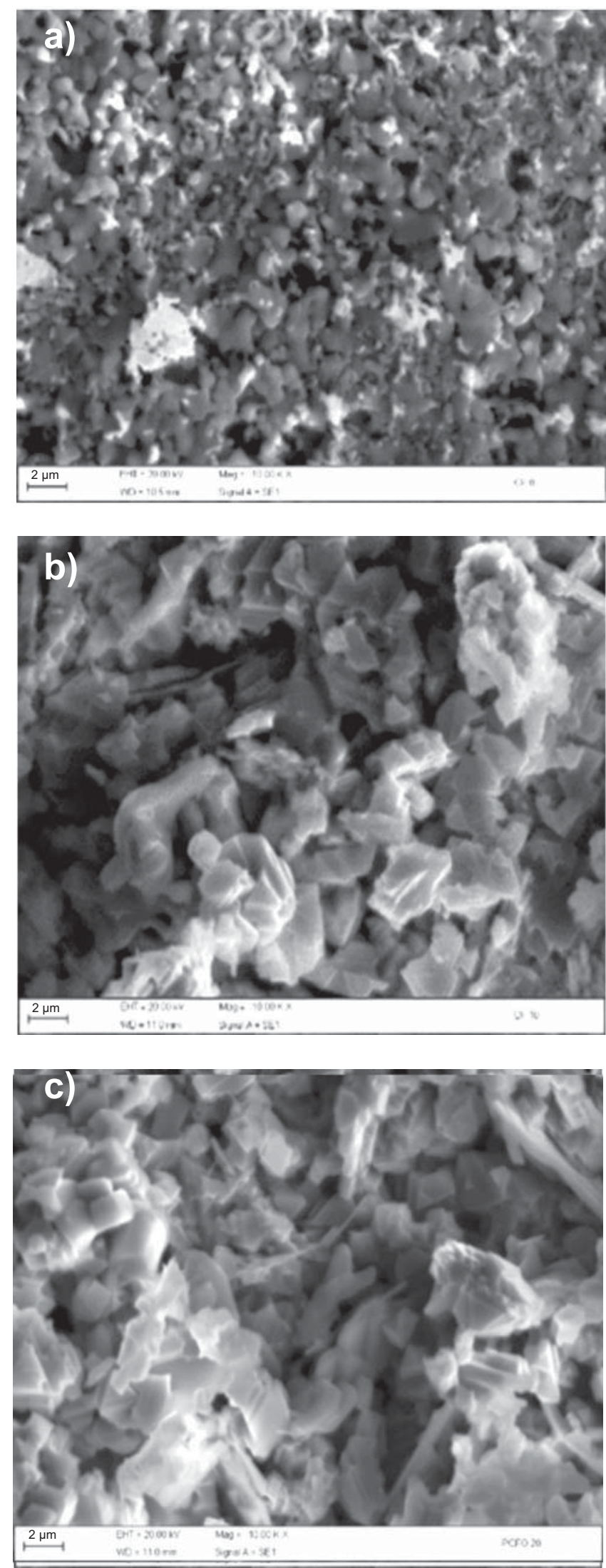

Figura 4: Micrografias das amostras sinterizadas. Imagens obtidas por $\operatorname{MEV}$ (a) $\mathrm{x}=0$, (b) $\mathrm{x}=0,10$ e (c) $\mathrm{x}=0,20$.

[Figure 4: Micrographs of sintered samples. Images obtained by $\operatorname{SEM}(a) x=0$, (b) $x=0.10$ e (c) $x=0.20$.] 
necessitando de um campo magnético muito alto para movimentar as paredes dos domínios.

As Figs. 4a a 4c mostram as micrografias das amostras $\mathrm{Pb}_{\mathrm{x}} \mathrm{Cu}_{(1-\mathrm{X})} \mathrm{Fe}_{2} \mathrm{O}_{4}(\mathrm{X}=0,0,1,0,2)$. Pode-se notar a tendência de formação de cristais finos para formar mini ou macroaglomerados com formas, tamanhos e a presença de poros irregulares. Observa-se que as amostras são formadas por partículas de tamanhos variados e com morfologia esférica. Observa-se também que a adição de chumbo levou a um aumento no tamanho médio das partículas, de 0,436-0,813 $\mu \mathrm{m}$ para a amostra isenta de chumbo $(\mathrm{x}=0)$ para $0,484-1,061$ $\mu \mathrm{m}$ para a amostra com estequiometria $\mathrm{x}=0,10$ de $\mathrm{PbO}$ e de 0,449-0,985 $\mu \mathrm{m}$ para a amostra com $\mathrm{x}=0,20$ de $\mathrm{PbO}$. O aumento do tamanho das partículas está relacionado com a adição de íons de $\mathrm{Pb}$ nas amostras. Quando se compara as amostras contendo 0,10 e 0,20 de $\mathrm{PbO}$, observa-se que não houve variação significativa no tamanho de partículas e nem no campo coercivo (Tabela II).

\section{CONCLUSÕES}

Os resultados mostraram que as amostras produzidas são formadas exclusivamente pela fase com a estrutura de espinélio com simetria tetragonal. $\mathrm{O}$ aumento da concentração de $\mathrm{PbO}$ proporcionou uma diminuição da porosidade e consequentemente aumento na densidade das amostras. A adição de $\mathrm{PbO}$ aumentou os valores de densificação a verde de $21,3 \%$ para $56,2 \%$, nas ferritas isentas de chumbo e contendo $\mathrm{X}=0,2$, respectivamente. $\mathrm{A}$ densidade aparente das amostras sinterizadas aumentou de $3,07 \mathrm{~g} / \mathrm{cm}^{3}$ para $3,70 \mathrm{~g} / \mathrm{cm}^{3}$, nas ferritas isentas de chumbo e contendo $\mathrm{X}=0,2$, respectivamente. As curvas de histerese possuem características de um material magnético mole. Essas curvas mostram também que a magnetização cresce com o aumento do campo magnético não ocorrendo a saturação magnética. Observou-se também que o aumento da concentração de chumbo aumentou a magnetização de saturação e diminuiu o campo coercivo e a remanescência das amostras. A dopagem com chumbo proporcionou também o aumento do tamanho das partículas e diminuição da porosidade o que contribuiu para a diminuição do campo coercivo das amostras e aumento da magnetização de saturação.

\section{AGRADECIMENTOS}

Os autores agradecem a CAPES, CNPq e FAPEMIG pelos apoios financeiros.

\section{REFERÊNCIAS}

[1] V.B. Gaikwad, S.S. Gaikwad, R.D. Nikam, "Synthesis and characterization of nano-crystalline $\mathrm{Cu}$ and $\mathrm{Pb}$ ", Sens. Transducers 134, 11 (2011) 132-142.

[2] R. Tebble, D.J. Craik, "Magnetic Materials", Willey Interscience (1969) 345.

[3] C. Heck, "Magnetic Materials and their Applications",
Butterworth (1974) 407.

[4] R.A. Torquato, F.A. Portela, L. Gama, D.R. Cornejo, S.M. Rezende, R.H.G.A. Kiminami, A.C.F.M. Costa, "Avaliação da microestrutura e das propriedades magnéticas de ferritas Ni-Zn dopadas com cobre", Cerâmica 54, 329 (2008) 55-62. [5] S.M. Hoque, M.S. Ullah, F.A. Khan, M.A. Hakim, D.K. Saha, "Structural and magnetic properties of $\mathrm{Li}-\mathrm{Cu}$ mixed spinel ferrites", Phys. B: Condens. Matter. 406, 9 (2011) 1799-1804.

[6] Q.M. Wei, J.B. Li, Y.J. Chen, Y.S. Han, "X ray study of cation distribution in $\mathrm{NiMn}_{1-\mathrm{x}} \mathrm{Fe}_{2-\mathrm{x}} \mathrm{O}_{4}$ ", Mater. Charact. 47 (2001) 247-252.

[7] J.D. Dunitz, L.E. Orgel, "Eletronic proprietes of transition metal oxides: Distortions from cubic symmetry", J. Phys. Chem. Solids 3 (1957) 20-29.

[8] Y. Mastai, Crystalization in Spinel Ferrite Nanoparticles, Adv. Crystallization Proc. (2012).

[9] K.S.R.C. Murthy, S. Mahanty, J. Ghose, "Phase Transition studies on copper ferrite", Mater. Res. Bull. 22 (1987) 1665-167.

[10] I. Xiao, A.S. Jin, A.Wang, A.W. Li , B.J. Wangc, C. Liang, "Preparation, structure and catalytic properties of magnetically separable $\mathrm{Cu}-\mathrm{Fe}$ catalysts for glycerol hydrogenolysis”, J. Mater. Chem. 12 (2012), 16598-16605.

[11] H. Yang, J. Yan, Z. Lu, X. Cheng, Y. Tang, "Photocatalytic activity evaluation of tetragonal $\mathrm{CuFe}_{2} \mathrm{O}_{4}$ nanoparticles for the $\mathrm{H}_{2}$ evolution under visible light irradiation, J. Alloy. Compoun. 476 (2009) 715-719.

[12] Z. Ping, Y. Bo, Z. Lei, "Mechanism of oxygen releasing of copper ferrite in the formation of the corresponding oxygen-deficient compound", Sci. China Series B 52, 1 (2009) 101-108.

[13] J. Plocek, A. Hutlová, D. Nižňanský, J. Buršík, J.L. Rehspringer, Z. Mička, "Preparation of $\mathrm{CuFe}_{2} \mathrm{O}_{4} / \mathrm{SiO}_{2}$ nanocomposite by the sol-gel method", Mater. Sci.-Poland 23, 3 (2005) 697.

[14] N. Rezlescu, L. Sachelarie, E. Rezlescu, C.L. Sava, P.D. Popa, "Influence of $\mathrm{PbO}$ on microstructure and properties of a NiZn ferrite", Ceram. Int. 29 (2003) 107-111.

[15] R.R. Corrêa, C.W. Pachoal, J.L.P. Dominici, "Preparação e caracterização da hexaferrita de bário", Cerâmica 59 (2013) 518-521.

[16] S.A. Mazen, "Tetravalent ions substitution in Cu-ferrite, structure formation and electrical properties", Mater. Chem. Phys. 62 (2000) 131-138.

[17] Z. Ullah, S. Atiq, S. Naseem, "Influence of Pb doping on structural, electrical and magnetic properties of Srhexaferrites", J. Alloy. Compoun. 555, 5 (2013) 263-267.

[18] O. Mirzaee, "Influence of $\mathrm{PbO}$ and $\mathrm{TiO}_{2}$ additives properties of Ni-Zn soft ferrites", J. King Saud University Eng. Sci. 26, 2 (2014) 152-58.

[19] T. Anjaneyulu, P.N. Murthy, S.K.M. Rafi, S.K. Bademiya, G.S. John, "Effect on magnetic properties of zinc doped nano ferrites synthesized by precursor or method", Int. Lett. Chem. Phys. Astron. 14 (2013) 37-43.

[20] M. Niyaifar, H. Shalilian, A. Hasanpour, H. Mohammadpour, "Effect of copper substitution on structural 
and magnetic properties of NiZn ferrite nanopowders", J. Magn. 18, 4 (2013) 391-394.

[21] A. Haq, M.A.U. Rehman, "Effect of Pb on structural and magnetic propertiesof Ba-hexaferrite", Phys. B: Condens. Matter. 407, 5 (2012) 822-826.

[22] L. Sachelarie, C. Doroftei, E. Rezlescu, "Microstructure and magnetic and electrical properties of low-temperature sintering Ni-Zn, $\mathrm{Li}-\mathrm{Zn}$ and $\mathrm{Mg}-\mathrm{Zn}-\mathrm{Cu}$ ferrites without and with addition of lead oxide", J. Optoelectron. Adv. Mater. 12, 4 (2010) 864-867.

[23] V.J. Da Silva, "Síntese por Reação de Combustão, Sinterização e Caracterização de Ferritas Ni-Zn Dopadas com Cromo", Universidade Federal de Campina Grande, Campina Grande (2007).

[24] R.A.M. Meneses, "Microestrutura e Propriedades Elétricas da Zircônia dopada com óxidos mistos de Terrras raras para aplicação como elétrolito sólido em sensores de oxigênio", Universidade de Brasília, Brasília (2013).

[25] L. Koshimizu, M.R. Morelli, "Adição de grafite na conformação de substratos cerâmicos porosos processados por rolos a frio", Cerâmica 58, 348 (2012) 516-520.

[26] K.H. Maria, S. Choudhury, M.A. Hakim, "Structural phase transformation and hysteresis behavior of $\mathrm{Cu}-\mathrm{Zn}$ ferrites", Int. Nano Lett. 42 (2013) 1-10.

[27] L. Vergard, "Die Konstitution der Mischkristalle und die Raumfüllung der”, Zeits Phys. 2 (1921) 17-26.

[28] Z. Xu, "Magnetic anisotropy and Mossbauer spectra in disordered lithium-zinc", J. Appl. Phys. 93 (2003) 47464749.

[29] S.K. Nath, K.H. Maria, S. Noor, S.S. Sikder, S.M. Hoque, M.A. Hakim, "Magnetic ordering in Ni-Cd ferrite", J. Magn. Magn. Mater. 324 (2012) 2116-2120.

[30] S. Noor, M.A. Hakim, S.S. Sikder, S.M. Hoque, K.H. Maria, P. Nordblad, "Magnetic behavior of $\mathrm{Cd}^{2+}$ substituted cobalt ferrites", J. Phys. Chem. Solid. 73 (2012) 227-231.

[31] S. Akhter, M.A. Hakim, "Magnetic properties of cadmium substituted lithium", Mater. Chem. Phys. 120 (2010) 399-403.

[32] P. Smitha, P.K. Pandey, S. Kurian, N.S. Gajbhiye, "Mossbauer studies and magnetic properties of spinel lead ferrite", Hyperfine Interact. 184 (2008) 129-134.

[33] R.H. Kadam, A. Karim, A.B. Kadam, A.S. Gaikwad, S.E. Shirsath, "Influence of $\mathrm{Cr}^{3+}$ substitution on the electrical and magnetic properties of $\mathrm{Ni}_{0.4} \mathrm{Cu}_{0.4} \mathrm{Zn}_{0.2} \mathrm{Fe}_{2} \mathrm{O}_{4}$ nanoparticles", Int. Nano Lett. 28 (2012) 1-5.

[34] M.V. Chaudhari, S.E. Shirsath, A.B. Kadam, R.H. Kadam, S.B. Shelke, D.R. Mane, "Site occupancies of $\mathrm{Co}-\mathrm{Mg}-\mathrm{Cr}-\mathrm{Fe}$ ions and their impact on the properties of $\mathrm{Co}_{0.5} \mathrm{Mg}_{0.5} \mathrm{Cr}_{\mathrm{x}} \mathrm{Fe}_{2-\mathrm{x}} \mathrm{O}_{4}$ ”, J. Alloy. Compoun. 552 (2013) 443450.

(Rec. 22/04/2015, Ac. 22/07/2015) 